\title{
Les trois vi-e-s des territoires ruraux : voyage en France ou ailleurs
}

\author{
Sylvie Lardon ${ }^{\mathrm{a}}$
}

«Des territoires vivants pour transformer le monde. »

(Caron et collab., 2017)

RÉSUMÉ. Les territoires ruraux changent : ils nouent de nouvelles relations avec le monde urbain. La planète s'urbanise : elle est le siège de nombreux flux. Ces dynamiques, qui s'étendent sur de larges espaces, occultent celles qui se déroulent à proximité, à faible densité, dans les territoires où pourtant on assiste à un foisonnement d'initiatives. Or, ce sont des territoires vivants qui transforment le monde en articulant l'urbain et le rural, en assumant une solidarité réciproque, en dépassant les limites et les frontières. Pour accompagner ces dynamiques de changement, l'ingénierie territoriale se doit de favoriser l'interterritorialité, l'intégration des échelles et des mondes ainsi que la reconnaissance des innovations sociales et territoriales. Plus encore, elle doit impliquer l'ensemble des acteurs qui sont partie prenante pour donner vie aux territoires en se construisant une vision partagée du territoire et en mettant en œuvre des actions collectives, en les ancrant dans le territoire et en les rendant visibles et sensibles. Ce cadre conceptuel d'une ingénierie territoriale pour, par et avec les territoires ruraux est issu d'une recherche partenariale en cours avec le territoire du Grand Clermont et du parc naturel régional Livradois-Forez, en région AuvergneRhône-Alpes, en France, qui « invente les territoires de demain » autour de son projet alimentaire territorial. Il y a là de nouvelles pistes à explorer qui remettent en cause les procédés traditionnels de production scientifique et de construction de l'action. La rigueur des itinéraires méthodologiques construits et la diversité assumée des dispositifs permettent de monter en généricité. L'imagination valorise cette diversité et produit le futur des territoires.

\begin{abstract}
Rural areas are changing: they are forging new relationships with the urban world. The planet is urbanizing: it is the seat of many flows. These dynamics, which extend over large spaces, obscure those that take place nearby, at low density, in territories where, however, we are witnessing a proliferation of initiatives. However, these are living territories which transform the world, by articulating the urban and the rural, by assuming a mutual solidarity, by crossing the limits and the borders. To support these dynamics of change, territorial engineering must promote inter-territoriality, the integration of scales and worlds, and the recognition of social and territorial innovations. But even more, it must involve all the stakeholders, to give life to the territories, by building a shared vision of the territory and implementing collective actions, by anchoring them in the territory, and by making them visible and sensitive. This conceptual framework of territorial engineering for, by and with rural territories is the result of ongoing partnership research with the territory of Grand Clermont and the Livradois-Forez. Regional Natural Park, in the Awvergne-Rhône-Alpes region, in France, which "invents the territories of tomorrow" around its territorial food project. There are new avenues to explore, which challenge the classic processes of scientific production and construction of action. The rigor of the methodological routes constructed and the assumed diversity of the devices allow us to build in genericity. The imagination values this diversity and produces the future of the territories.
\end{abstract}

\section{Introduction}

Les territoires ruraux changent, bougent et se transforment. Ils nouent de nouvelles relations avec le monde urbain. La planète s'urbanise : elle est le siège de nombreux flux, économiques, d'informations, de migrants qui cherchent d'autres lieux pour vivre. Les mobilités sont voulues (loisirs, tourisme, découverte du monde) ou subies (guerres, risques naturels, insécurité). La mondialisation est là, avec son lot d'avantages (connaissance d'autres cultures, développement des technologies) et d'inconvénients (diffusion des épidémies, homogénéisation des produits).

\footnotetext{
a Institut national de la recherche pour l'agriculture, l'alimentation et l'environnement (INRAE) et AgroParisTech, UMR Territoires, Clermont-Ferrand, France
} 
Ces dynamiques, qui s'étendent sur de larges espaces, occultent celles qui se déroulent à proximité, à faible densité, dans les territoires où pourtant on assiste à un foisonnement d'initiatives. «En définitive, dans une société urbaine mondialisée, la campagne est peut-être en meilleure posture pour imaginer de nouvelles figures du local permettant d'opposer à la banalisation des lieux et des modes de vie des formes d'expression très variées.» (Margetic, Roth et Pouzenc, 2017, p. 7) Allons à la rencontre de quelques-unes d'entre elles pour comprendre ce qui se passe dans les territoires ruraux :

Les formes territoriales émergentes peuvent être considérées comme autant de prémices de nouveaux modèles d'organisation apportant des réponses à un problème particulier et valorisables, éventuellement dans une perspective de développement durable, à une échelle plus large. (Caron, 2017, p. 18)

Le territoire, en ce qu'il permet d'innover et de renforcer le contrôle des transformations par les acteurs, est un maillon essentiel pour concevoir et expérimenter de nouvelles formes d'intervention adaptées à chaque situation. En ce qu'il connecte actions publique et collective, il l'est également pour articuler actions locales, nationales et internationales et pour jouer le premier rôle d'une ingénierie multiscalaire du développement durable. (Valette, Caron, Coppens d'Eeckenbrugge et Wassenaar, 2017, p. 272)

C'est avec ce parti-pris d'une approche territoriale que nous abordons ce voyage au sein des territoires, en France ou ailleurs, à la rencontre des ruralités contemporaines. C'est dans une perspective de développement territorial, où les acteurs se donnent la capacité de maitriser les processus qui les concernent (Deffontaines, Marcelpoil et Moquay, 2001), que nous tirons les leçons de diverses expériences vécues ou racontées, tant les récits de vie expriment les dynamiques non encore advenues qui feront le futur des territoires.

Dans une première partie, nous présenterons les diverses façons d'entrer en contact avec les territoires ruraux, pour une chercheuse elle-même investie dans le développement territorial. Puis, au fil d'expériences racontées, dans différents lieux et dans diverses circonstances, nous déroulerons progressivement quelques fils pour comprendre ce qui est en train de se passer dans les territoires ruraux, ici et ailleurs. Enfin, par un retour réflexif sur ce que nous avons vu et compris des territoires, nous rendrons compte des trois vi-e-s des territoires ruraux, attachées aux trois verbes vouloir, vivre et voir, d'une approche pour accompagner les « territoires vivants pour transformer le monde », une nouvelle ingénierie territoriale qui dessine les territoires (Debarbieux et Vanier, 2012) et qui invente les figures du projet territorial (Debarbieux et Lardon, 2003).

\section{1. À la rencontre des territoires ruraux par des voies détournées}

Pour un chercheur, fût-il en sciences humaines et sociales, la voie d'entrée traditionnelle dans la complexité des systèmes humains est l'analyse documentaire, l'entretien de terrain et le croisement des données. Sans trop nous en éloigner, nous avons préféré un chemin détourné : a) par les documents vivants, ceux des témoignages d'acteurs et de chercheurs lors de colloques un peu décalés, b) par des ateliers de coconstruction, supports de paroles d'acteurs, et c) par des regards croisés de chercheurs, a posteriori. Nous avons ainsi nommé les trois façons d'entrer dans le vif du sujet et d'apprendre par la découverte du monde des objets (Bonnard, 2015).

\section{a) Les documents vivants: témoignages d'acteurs et de chercheurs lors de colloques}

La première façon est somme toute assez traditionnelle pour un chercheur : il s'agit de se nourrir des connaissances partagées lors de colloques ciblés sur sa problématique. Or, quand le colloque s'intitule "Faire monde commun", qu'il se passe dans un lieu chargé d'histoire (soit le musée de Bibracte, une ville gallo-romaine), qu'il fait se rencontrer des chercheurs et des acteurs du territoire (du parc naturel régional du Morvan en région BourgogneFranche-Comté (CESER Bourgogne-FrancheComté, 2017)), il se passe des choses inattendues. Quand c'est un séminaire chercheur-acteur du projet de recherche INVENTER - Inventons nos territoires de demain ${ }^{1}$ et qu'il se fait itinérant pour combiner témoignages d'acteurs et propos de chercheurs, il y a des connexions qui se font. Quand ce sont les acteurs eux-mêmes, accompagnateurs des projets de territoire (p. ex., la Plateforme 21 pour le développement durable ${ }^{2}$ ou CapRural ${ }^{3}$ en 
Auvergne-Rhône-Alpes), qui organisent le dialogue avec les chercheurs, il y a une prise de recul collective qui s'opère, puis chacun repart enrichi d'expériences nouvelles.

\section{b) Les ateliers de coconstruction}

La seconde façon est proactive et s'inscrit dans une longue perspective de recherche-formation-action, dans laquelle :

La formation est conçue comme une interface entre chercheurs et acteurs du développement territorial et comme moteur de leurs interactions. La formation est un médiateur entre la recherche et l'action, au sens où elle intervient dans l'action en se plaçant comme interlocutrice des questions de développement portées par les acteurs et où elle intervient dans la recherche en interpellant les chercheurs sur les concepts et les méthodes à produire pour faciliter l'apprentissage collectif. Elle mobilise et génère de nouvelles connaissances; elle s'appuie sur et favorise l'acquisition de compétences. » (Lardon et collab., 2015, p. 48)

Nous avons nommé le dispositif «jeu de territoire », mis au point en 2003 lors d'un séminaire de recherche (Angeon et Lardon, 2003), testé en 2004 avec les acteurs du territoire au moment de la construction du viaduc de Millau (Lardon, Moquay et Poss, 2007). Ensuite, nous l'avons stabilisé dans un autre contexte, celui d'une région éloignée du Québec, au Témiscamingue (Lardon, 2013). Depuis lors, nous avons «joué » plus de 50 fois dans des situations variées.

L'approche repose sur trois principes (spatialisation, participation des acteurs et portage politique) et partage avec l'ensemble des acteurs qui sont partie prenante. Le jeu de territoire se joue en trois étapes (diagnostic, prospective et pistes d'action) pour construire une vision partagée du territoire et de ses enjeux, et pour impliquer les acteurs dans des actions collectives pour leur projet de territoire.

\section{c) Les regards croisés de chercheurs}

La troisième façon est indirecte : elle se fait par l'accompagnement de jeunes chercheurs pour les aider à construire leur propre itinéraire méthodologique de recherche, dans des ateliers que nous avons organisés ces dernières années au sein de l'Unité mixte de recherche Territoires ${ }^{4}$. Au fil des exposés des jeunes chercheurs, l'approfondissement méthodologique permet aussi de rapidement s'intéresser à différentes histoires, qu'on peut faire siennes et valoriser dans d'autres circonstances.

C'est avec cette richesse de points de vue que nous pouvons entrer dans les dynamiques des territoires ruraux et regarder le monde autrement.

\section{2. À la croisée des chemins, « une autre vie s'invente ici » (PNR, 2007)}

Il ne s'agit pas ici d'approfondir quelques études de cas, ni même de les comparer et d'évaluer leurs apports réciproques. Il s'agit plutôt d'évoquer quelques expériences pour les reconnaître comme de potentiels leviers de développement territorial (Loudiyi et Houdart, 2019). Si elles sont souvent fédérées autour d'une problématique commune, celle de l'alimentation (Lardon et Loudiyi, 2014), elles peuvent prendre racine dans d'autres enjeux du développement territorial, qu'ils soient économiques, sociaux, environnementaux ou culturels (Torre et Wallet, 2015).

\subsection{Des articulations inédites}

Le territoire du Grand Clermont et du parc Livradois-Forez fait l'objet d'un projet alimentaire territorial labellisé en 2017 dans le cadre du Programme national pour l'alimentation ${ }^{5}$ :

Un PAT constitue un ensemble d'initiatives locales, coordonnées dans le cadre d'une stratégie territoriale globale et transversale visant à développer un système alimentaire territorial et à structurer l'économie agro-alimentaire par le rapprochement des acteurs impliqués dans ce système et le développement d'une alimentation locale, durable et de qualité. (DRAAF Auvergne, 2015, p. 1)

Les acteurs de ce même territoire, le Grand Clermont et le parc Livradois-Forez, sont partenaires du programme de recherche-action INVENTER - Inventons nos territoires de demain 2015-2020 :

Le projet INVENTER vise à formaliser les dynamiques d'évolution des territoires ruraux et métropolitains, en prenant appui sur la gou- 
vernance alimentaire, et à concevoir l'accompagnement du changement dans un dispositif de recherche-formation-action. Pour ce faire, les chercheurs d'INVENTER s'intéressent aux territoires de projet, où s'inventent de nouvelles façons d'alimenter et d'accompagner les territoires, tant sous l'impulsion de politiques territorialisées que d'actions collectives. Le projet a donc l'ambition d'inventer de nouveaux modèles de développement territorial, dans un partenariat interactif et créatif, entre chercheurs et acteurs, à différentes échelles d'action. (INRA, 2015, s. p.)
De ce fait, le PAT du Grand Clermont et du parc Livradois-Forez a été à la fois l'objet d'étude du projet de recherche-action INVENTER et le lieu d'expérimentation de nouvelles modalités d'articulation urbaine-rurale.

Plusieurs jeux de territoire y ont été menés. En 2016, le jeu «Inventons notre territoire de demain » (Trimech et Lardon, 2017a), mené sur le grand territoire, a mis en évidence l'enjeu, pour les acteurs locaux, d'un même modèle de développement, tant pour la partie urbaine (Grand Clermont) que pour la partie rurale (parc Livradois-Forez). Le scénario combine points de vente locaux, ateliers de transformation et éducation alimentaire des enfants sur tout le territoire (voir Figure 1).

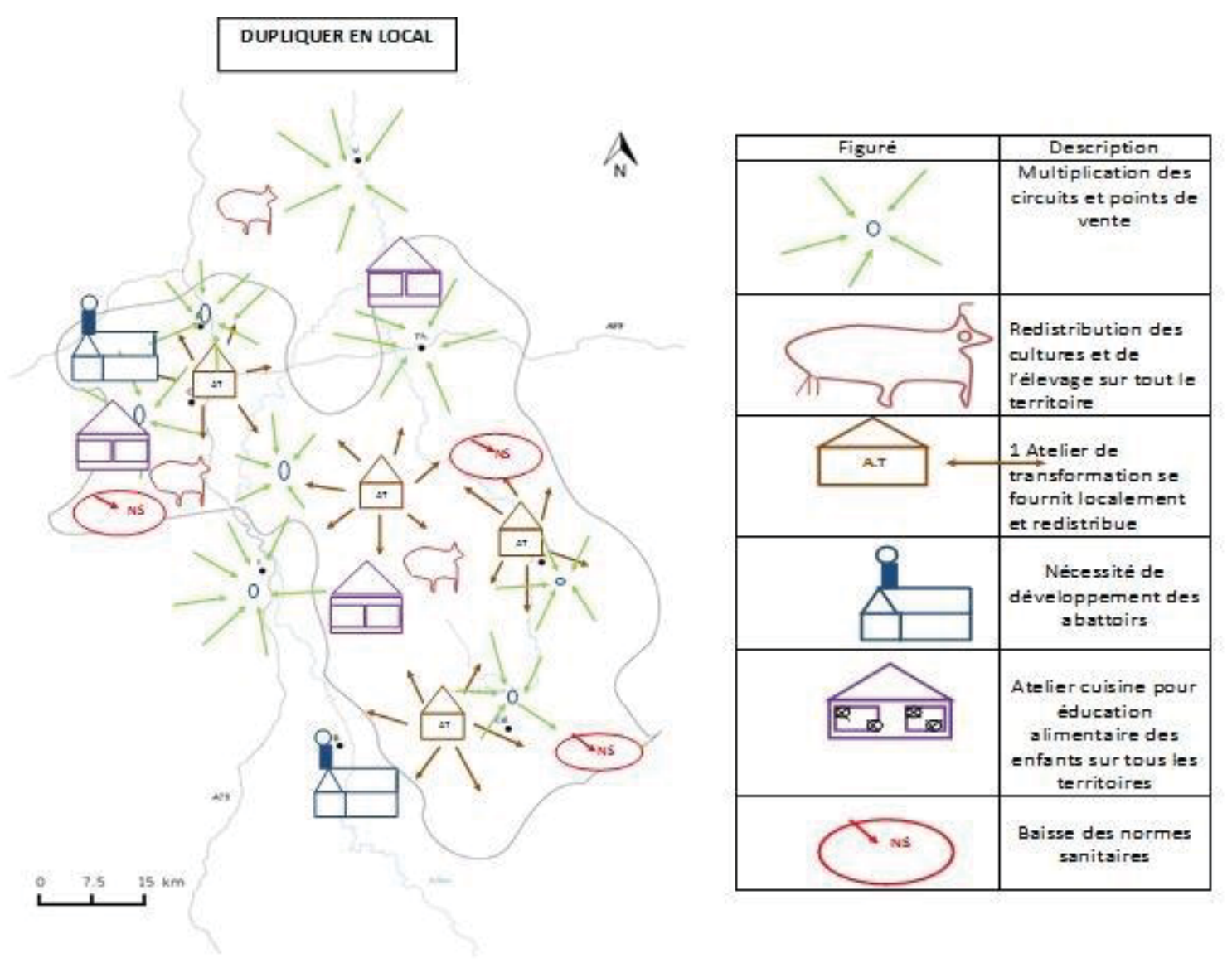

Figure 1 - Scénario d'évolution du territoire du Grand Clermont et du PNR Livradois-Forez Source : Trimech et Lardon, 2017a 
Le deuxième jeu, "Inventons notre alimentation de demain » (Trimech et Lardon, 2017b), a été mené en 2017 à l'échelle de Billom Communauté, un regroupement de communes, charnière entre le Grand Clermont et le parc Livradois-Forez. Il a concrétisé ce modèle de développement. Dans le scénario d'évolution proposé par les acteurs, le centre de distribution alimentaire, localisé à Billom, articule les dynamiques de polarisation vers le Grand Clermont, sur la partie ouest du territoire, avec les dynamiques de constitution d'une ceinture verte, dans la partie plus rurale du parc Livradois-Forez, à l'est. Ainsi, la différenciation du territoire agricole est valorisée et l'approvisionnement en produits locaux est possible pour tous (voir Figure 2).

Ces travaux de recherche, antérieurs au plan d'actions alimentaires élaboré en 2018, illustrent bien la volonté des acteurs locaux de coordonner les actions, fussent-elles hétérogènes, et d'articuler les échelles, du local au grand territoire. La mise en œuvre du projet alimentaire, en cours, répond effectivement à cette double exigence. C'est ainsi que plusieurs initiatives, souvent lancées en amont, prennent une nouvelle ampleur par leur reconnaissance par les acteurs institutionnels et professionnels. Les magasins de producteurs, tels que le magasin Le Local à Ambert (Iceri, 2019) et le magasin Aux Champs à Thiers, sont des lieux de valorisation de produits locaux, mais aussi de réponse à des enjeux sociaux et territoriaux (rénovation des centres-bourgs à Ambert, territoire zéro chômeur à Thiers). L'usage de la monnaie locale, la doume, initialement portée par les acteurs de la société civile (Houdart et Nguyen Ba, 2018), prend de l'ampleur au sein du département et impulse le soutien de la métropole ClermontAuvergne pour le développement de filières alimentaires localisées. Le réseau des jardiniers, idée issue du jeu de territoire de Billom Communauté, a vu le jour en 2019 (Beauseroy, Malbon, Mariotti, Lalanne et Lardon, 2019; voir Figure 3). Ce sont autant de témoignages d'acteurs et d'analyses de chercheurs répertoriés lors du séminaire itinérant organisé par le projet INVENTER en juin 2019.

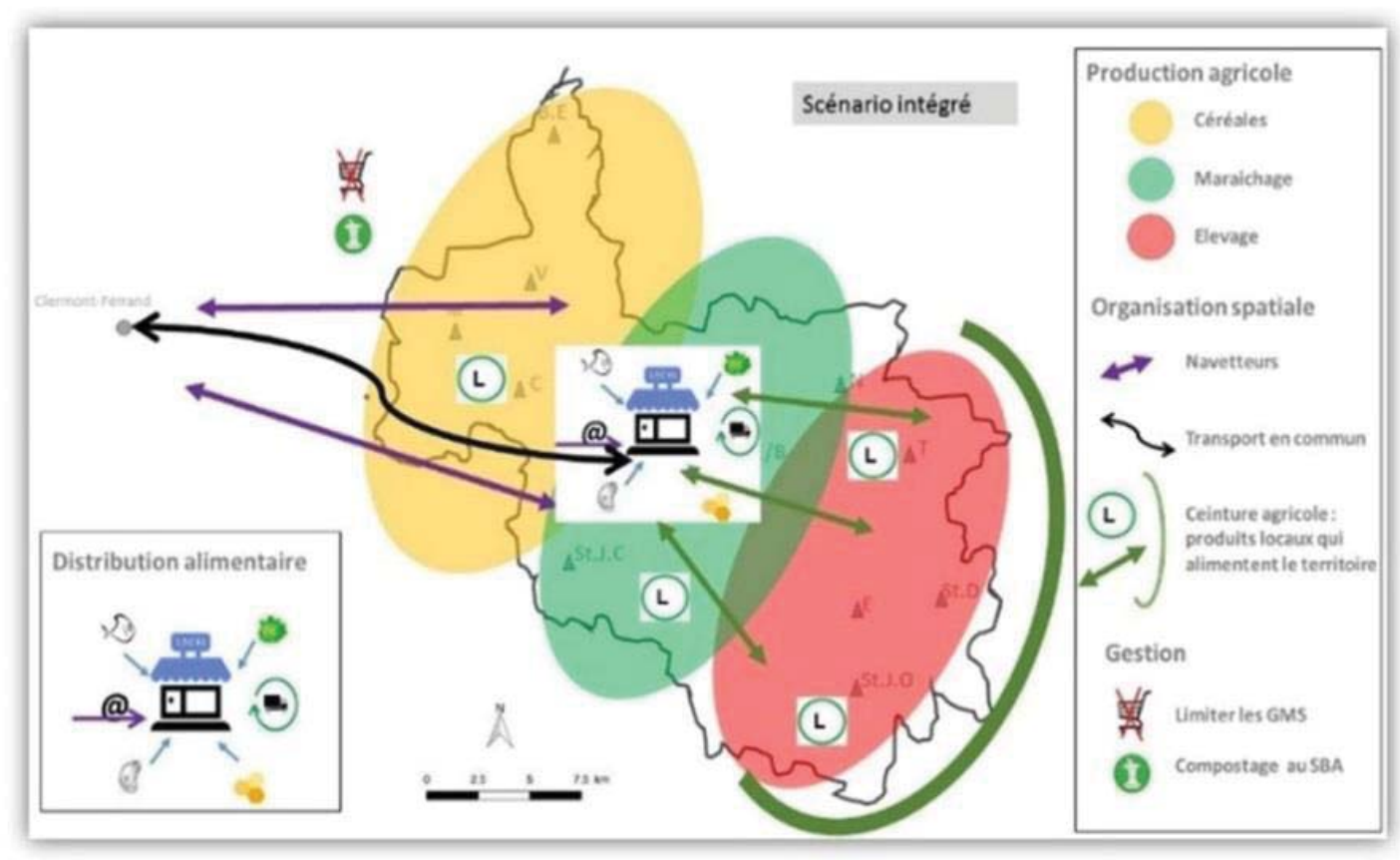

Figure 2 - Scénario d'évolution du territoire de Billom Communauté Source : Trimech et Lardon, 2017b 
Des espaces aménagés et appropriés

\section{Jardins}

Diversité de formes, gestion foncière, parcellaire, superficie, aménagements

La biodiversité, le climat

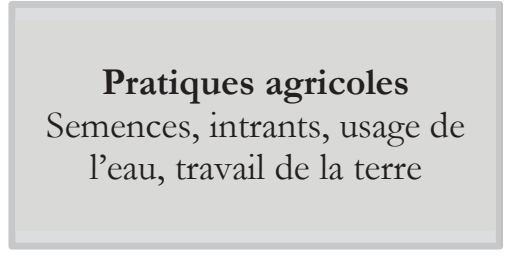

Une diversité de pratiques
Le jardinage social

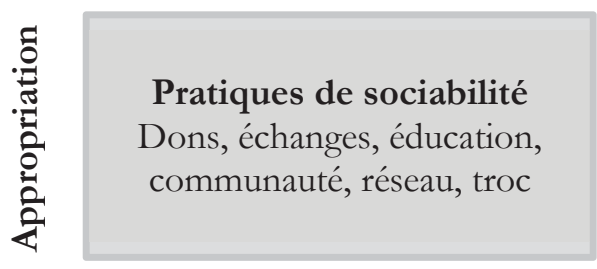

\section{La culture}

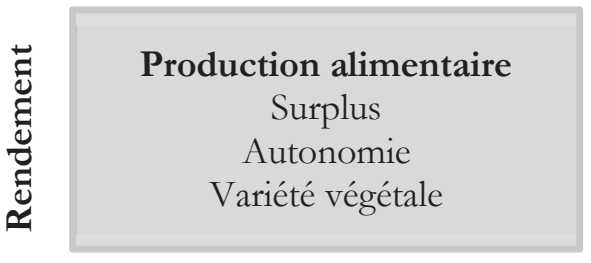

Des espaces de production

Figure 3 - Diversité des jardins et des jardiniers Source : d'après Beauseroy et collab., 2019

Or, réciproquement, l'expérience des acteurs techniques et politiques du projet alimentaire les amène à élargir leurs champs d'action commune, tout d'abord aux problématiques énergétiques et environnementales, en coordonnant sur le grand territoire les plans climat-air-énergie territoriaux (PCAET) des intercommunalités, puis aux questions de mobilité, dans un contrat de réciprocité avec Clermont Métropole. Il est à noter que ces initiatives ne sont actuellement connues que parce qu'il y a une arène locale, en l'occurrence le projet de recherche INVENTER, qui permet les échanges d'informations et la mise en synergie des actions entre chercheurs et acteurs. Cette façon d'identifier les signaux faibles des dynamiques en cours n'est pas à négliger. Heureusement, les acteurs eux-mêmes l'ont anticipé en proposant aux chercheurs de pérenniser leur collaboration en « instituant» un comité scientifique au sein même de la gouvernance du projet alimentaire. C'est une action en cours prometteuse, qui signe un apprentissage réciproque des chercheurs et des acteurs issus de leur confrontation, voire de leurs controverses (Lièvre, Johany et Nguyen Ba, 2019).

\subsection{Des solidarités assumées}

Les $14 \mathrm{e}$ entretiens de Bibracte-Morvan se sont déroulés du 11 au 13 septembre 2019. Ils étaient organisés par le parc du Morvan et le Centre archéologique européen de Bibracte, avec les chercheurs du Groupe de recherche Innovation et territoires de montagne de l'Université de Grenoble. Nous étions, chercheurs et acteurs, invités à « faire monde commun : venez participer à ce qui vous attache à votre territoire, afin d'y mieux vivre» (carton d'invitation, voir Figure 4).

\section{Comment définir ensemble ce qui fait la singularité de la vie en territoires de montagne pour esquisser un monde commun? \\ Venez participer aux discussions, donner votre avis, décrire votre vie! La manifestation faire monde commun propose une expérience ouverte à tous. Des regards, des imaginaires et des savoirs pratiques, scientifiques et techniques se croiseront autour de cette question.}

Figure 4-Extrait du carton d'invitation 
C'est ainsi que nous avons vécu une expérience unique, qui a secoué nos propres convictions de chercheuse et nos interrogations de citoyenne, en ouvrant de nouvelles fenêtres sur le monde. Il y avait tout à la fois le cadre théorique des communs (Orstrom, 2009), mais dans une acception territorialiste (Magnaghi, 2014), instrumenté par le sociologue Bruno Latour (2017) et ses étudiants de science politique à partir de son ouvrage Où atterrir? Il y avait une table ronde sur la montagne, sur l'hyper-rural et sur la marge pour croiser les regards et débattre (Depraz, 2017; De La Soudière, 2017), des mini-films documentaires et un long métrage sur les habitants du lieu. Des ateliers prospectifs ont été tenus pour imaginer l'agriculture de montagne sur les territoires du Grand Site BibracteMont Beuvray et des ateliers d'expression en commun sur les enjeux du territoire (gestion de l'eau en pleine sécheresse liée au changement climatique, gestion de la forêt avec les conflits autour des essences feuillues ou conifères, maintien d'activités dans une zone de montagne excentrée du Massif central, etc.). Le tout, dans des lieux insolites, avec méchoui à la ferme, ateliers au musée de Bibracte, logement en gîtes communaux, cantine au Centre archéologique européen et conférences à la salle polyvalente. Les participants étaient ralliés en covoiturage spontané, ce qui invitait à la spontanéité et à la créativité. Nous en retenons la grande force qui émane de dynamiques individuelles transformées par le collectif et la possibilité de faire monde commun malgré les différences, voire en combinant des contraires, dans un élan de solidarité.

On retrouve des solidarités assumées entre territoires différenciés, telles qu'on a pu les voir entre l'urbain et le rural pour le projet alimentaire du Grand Clermont et du parc Livradois-Forez, dans d'autres lieux et avec d'autres configurations. Ainsi, le territoire Guillestrois-Queyras, impliqué également dans un projet alimentaire porté par le parc du Queyras et le territoire du Briançonnais, des Écrins, du Guillestrois et du Queyras, articule montagne et vallées, espaces de nature et espaces agricoles (pâturages), production de lait valorisée par des fromageries locales en cours de constitution d'une appellation (Bleu du Queyras) et production de viande vendue en direct à la ferme grâce à l'abattoir de Guillestre géré en commun par les producteurs et les collectivités territoriales. Les habitants permanents et les touristes saisonniers sont attachés aux produits locaux et/ou bio; fréquentent les marchés locaux et les petites épiceries locales pour maintenir un maillage de commerces. Les habitants entretiennent leur jardin et leur poulailler pour une consommation autonome, et font preuve de solidarité envers les plus démunis (associations). Bien sûr, l'approvisionnement se fait encore beaucoup par la grande distribution, surtout dans ce contexte de difficiles mobilités liées au relief montagnard, mais les voisins s'organisent, des relais locaux sont trouvés, les déplacements administratifs et professionnels sont mis à profit (voir Figure 5).

Lors du jeu de territoire (Bletterie et Lardon, 2019), les participants avaient encore d'autres idées, comme la mise en place d'un glanage organisé : une structure associative organisée à l'aide d'une charte mettrait en relation des glaneurs et des propriétaires et exploitants agricoles pour procéder à la collecte des denrées alimentaires non récoltées (vergers non exploités, restes après récolte, invendus, cueillette) ou le financement d'un «transformobus» (bus itinérant qui parcourt tout le territoire pour faire des ateliers de transformation) afin de mieux conserver les produits locaux et de créer un lien entre les habitants (conservation en groupe, ateliers, etc.). Depuis, de nouvelles initiatives ont vu le jour, sous l'impulsion des acteurs institutionnels, qui se sont alliés dans une structure associative, le Média des Acteurs $^{6}$, pour organiser des ateliers participatifs, des forums citoyens, etc. 


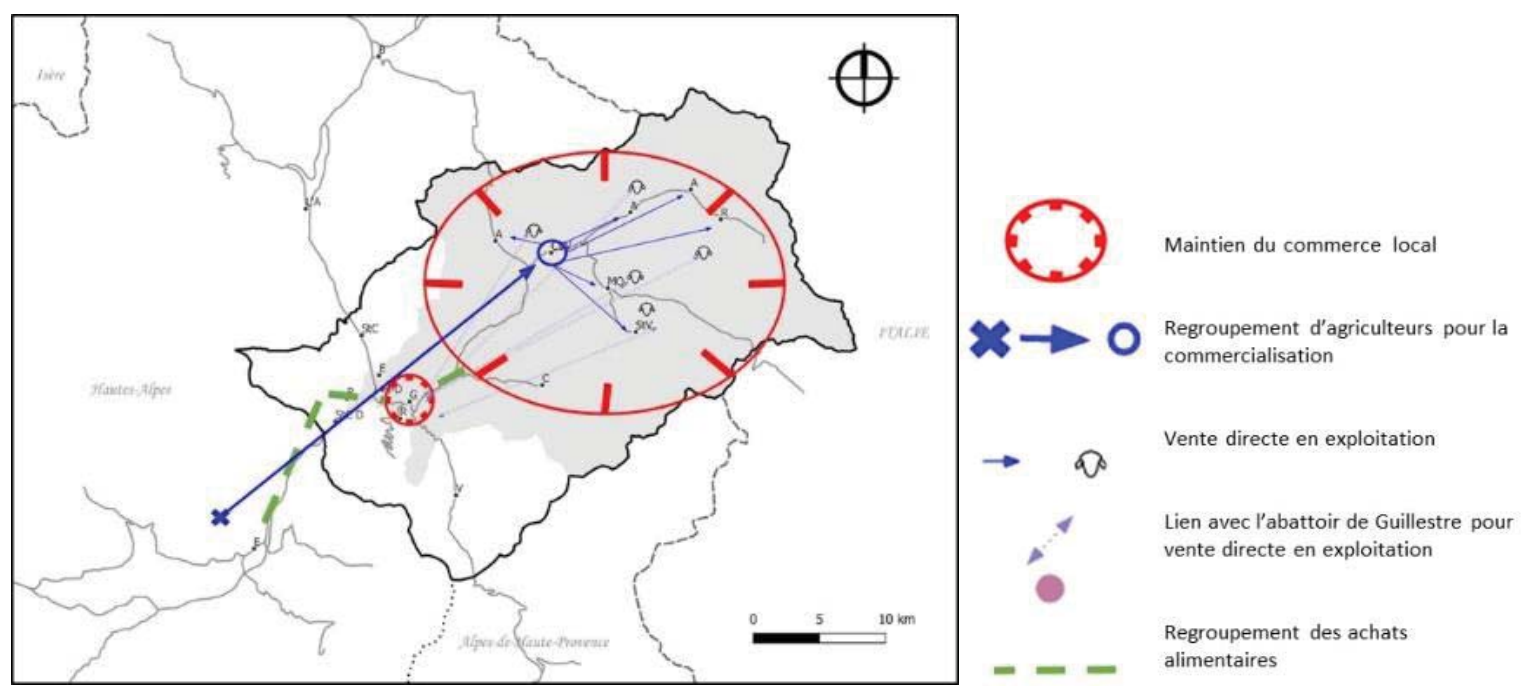

Figure 5 - Des territoires où il est possible d'être acteur de sa consommation alimentaire Source : Bletterie et Lardon, 2019

Dans la vallée de la Levrière, c'est aussi le contraste entre vallée et plateaux qui fait débat, et qui a incité le Conseil d'architecture, d'urbanisme et d'environnement de l'Eure ${ }^{7}$ et l'Association de la Vallée de la Levrière, dans le cadre du programme Paysages en transitions territoriales, à monter le projet Vallées habitées pour redynamiser la vallée. C'est dans ce cadre que nous avons mené un jeu de territoire (Lardon, Marraccini, Rizzo et Benoit, 2019b) visant à imaginer des circuits pour la vallée de la Levrière d'ici 2040.

Afin de mieux révéler les caractéristiques partagées de la vallée de la Levrière, il est vite apparu important pour les acteurs d'intégrer ses dynamiques économiques et ses activités agricoles à une échelle plus large, en prenant en compte la vallée de l'Epte, constitutive d'une même entité paysagère, et la ville de Gisors, qui structure le territoire. Le territoire des vallées de la Levrière et de l'Epte représente un bassin de production agricole important et diversifié (élevage de porcs ou de bovins [lait et viande], pisciculture, cultures [betterave, lin, colza, luzerne, orge, blé, etc.], vergers, exploitation forestière, héliciculture). Toutefois, ces produits peinent à être valorisés localement, malgré la présence d'industries agroalimentaires : une boulangerie-fromagerie artisanale, une sucrerie et une usine de déshydratation. Un des freins à un potentiel développement local semble la forte ouverture vers l'extérieur : forte tendance à l'exportation (demande essentiellement extérieure et, notamment, importants bassins de consommation à Rouen et Paris) et services de transformation situés à l'extérieur de la vallée, comme c'est le cas pour le service d'abattage.

L'esquisse d'un circuit local est à relever : quelques productions sont en vente directe, mais restent très fortement concurrencées par les supermarchés de Gisors. Le marché de Gisors est aussi touché par le phénomène d'attraction de ces établissements, et observe une nette baisse de fréquentation et une faible représentation des produits locaux. Finalement, ces initiatives, encore timides et isolées, sont le fait d'un potentiel manque de perspective de rentabilité à l'échelle de la vallée en raison de la faible densité démographique, d'un pouvoir d'achat contrasté, mais aussi du manque de mise en réseau et de structuration (en commercialisation) des différents producteurs et éleveurs locaux, qui ne parviennent pas à concurrencer l'offre extérieure des grands distributeurs ni à créer une identité particulière à leurs produits.

Ce sont ces problèmes que les acteurs ont tenté de résoudre. Deux thèmes abordés et particulièrement essentiels pour certains acteurs sont le lien social et l'accès aux services. En effet, le manque de services au sein de la vallée est très rapidement apparu comme un enjeu incontournable sur lequel travailler. (L'idée de marché fixe et mobile a été discutée.) De plus, les acteurs ont fait part d'une volonté de renforcer le lien social à travers l'aménagement de places publiques pour en faire des lieux de rencontre et de convivialité. Une autre réflexion transversale a été la volonté d'une piste à circulations 
douces et écologiques (vélos, trottinettes électriques, transports en commun électriques) reliant les différents villages et longeant le ruisseau de la Levrière. Ce sont autant d'actions qui sont lancées localement ${ }^{8}$.

\subsection{Des frontières dépassées}

Le Béarn et la Bigorre sont deux régions naturelles des Pyrénées, à cheval sur deux départements et deux régions administratives, La NouvelleAquitaine et l'Occitanie. Or, l'espace de vie TarbesLourdes-Pau (TLP) réunit 13 intercommunalités dont les enjeux communs dépassent les limites administratives mentionnées et nécessitent de les aborder dans des espaces cohérents, des nouveaux territoires ou des territoires de projets. Il s'agit des dynamiques du quotidien, qu'on retrouve dans les modalités de vie des habitants de cet espace : les multiples déplacements (travail, loisirs, familiaux, etc.), les activités collectives spécifiques (sportives, événementielles, etc.), les projets divers et les incitatifs locaux réalisés par des acteurs économiques (entreprises, artisans, etc.), la création d'associations, etc.

Cependant, il existe un fort enjeu sur le plan politique qui relève de la coopération/coordination de l'action publique dans ce périmètre. Cela pose les bases d'une problématique historique, qui empêche de développer certaines initiatives transfrontalières et d'atteindre le grand potentiel du Béarn-Bigorre, ce qui entraîne des conséquences négatives sur l'attractivité de cet espace en France et d'ailleurs ainsi qu'une forte dépendance croissante vis-à-vis des territoires voisins, notamment des deux grandes métropoles, Toulouse et Bordeaux.

C'est dans ce contexte que la Compagnie d'Aménagement des Coteaux de Gascogne (CACG) a lancé une démarche, à travers l'implication et l'engagement des acteurs locaux, afin d'identifier ensemble les enjeux communs et de favoriser l'action collective pour le développement des territoires du Béarn-Bigorre, avec un esprit ouvert à la participation de tous ces acteurs locaux, les habitants d'ici, qui se sentent prêts à agir pour coconstruire l'avenir.

Le jeu de territoire (Copello, 2019) organisé par la CACG et AgroParisTech a donné à voir l'organisation spatiale du territoire, avec le contraste sudnord entre le relief pyrénéen, son versant riche en ressources hydrologiques, la zone d'élevage pastoral en montagne et les prairies cultivées dans le piémont et le plateau céréalier, zone caractérisée par la qualité du sol, qui permet de cultiver le maïs. L'axe est-ouest, qui passe de Tarbes à Pau, concentre les déplacements entre pôles d'emplois. On observe finalement un effet miroir entre les deux parties du territoire, l'axe de symétrie étant la frontière administrative.

Un des scénarios imaginés est centré sur la mobilité et l'énergie (voir Figure 6). Les vallées sont reliées par une mobilité douce (transports collectifs de type tramway) et sont alimentées par des systèmes de production énergétique locaux (SPEL) autour de la méthanisation, de l'hydrogène et de l'hydroélectricité. Il y a des zones de production autonomes reliées, avec la possibilité de transfert d'énergie, qui bénéficient de la mobilité douce (tramway). Dans la zone montagne, une infrastructure transvallée, avec des vélos électriques ou à hydrogène, relie les vallées. Pour répondre aux problématiques des changements climatiques, les stations de ski sont reprises pour développer le vélo. Toujours en réaction aux changements climatiques, la rareté de la ressource en eau pose l'impératif de devoir faire des réserves d'eau pour l'agriculture ou, plus généralement, pour la population et les entreprises. Il y a une spécialisation des vols à l'international à l'aéroport Ossun-Lourdes et des vols nationaux à l'aéroport de Pau, avec des avions électriques ou à hydrogène à mobilité douce, moins polluants. Cet espace devient un même territoire politicoadministratif, porté politiquement par les pôles ruraux-urbains, et non par les agglomérations. 


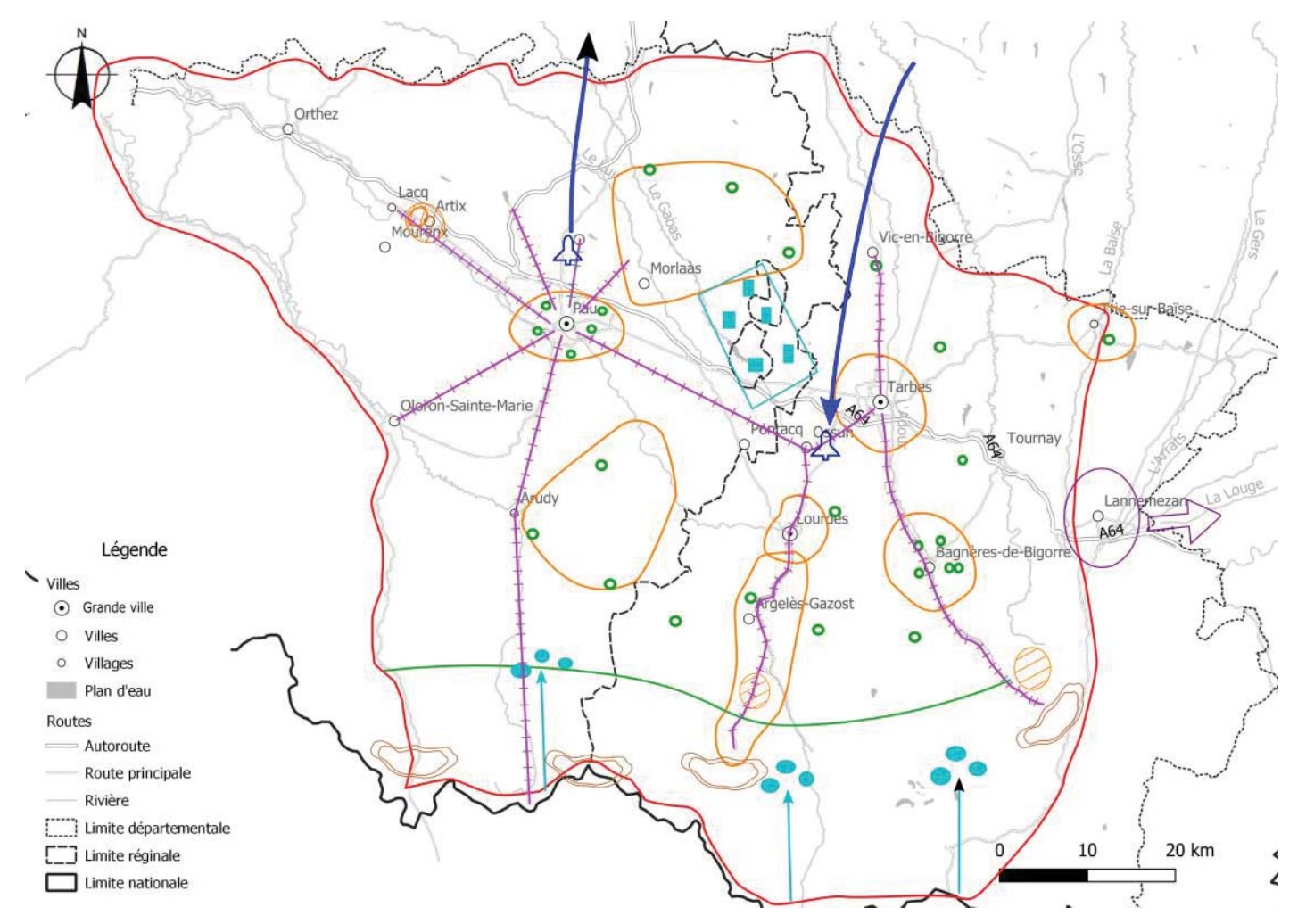

Figure 6 - Territoire d'innovation du pays Béarn-Bigorre Source : Copello, 2019

La technologie devient un facteur d'intégration territoriale, comme en d'autres lieux d'autres dynamiques innovantes. À Pise, en Italie, la préservation des terrasses en oliveraies du mont Pisano est le support d'une route de l'huile qui valorise les produits locaux. Les canaux de drainage de l'eau du mont sont aussi les canaux d'irrigation de l'agriculture céréalière de la plaine inondable de Pise. Les forêts littorales du parc naturel accueillent aussi les grandes exploitations expérimentales du parc et de l'université pour le développement des races bovines locales. Ces dynamiques confortent les projets alimentaires de la zone périurbaine de Pise (Filippini, 2015).

À Chascomús, en Argentine, le lien entre la ville et la campagne se recrée à travers l'organisation collective des producteurs. Le maintien de l'activité en milieu rural passe par une amélioration des infrastructures et par la préservation de l'environnement. On est loin d'une vision dichotomique de l'espace entre les grandes haciendas relevant d'un modèle productif intensif et les nouveaux projets d'agritourisme. L'agriculture et l'alimentation se combinent aux autres activités du territoire (Lardon, Copello et Albaladejo, 2019a).

\section{L'ingénierie territoriale au prisme des trois vi-e-s des territoires ruraux}

Toutes ces expériences relatées, tout comme beaucoup d'autres qui se déroulent sous nos yeux ici et ailleurs, montrent qu'il se passe quelque chose dans les territoires qui pourrait transformer le monde. Au-delà des processus globaux d'urbanisation, de mondialisation et des effets des changements climatiques, se déclinent, à l'échelle du territoire, des initiatives aussi variées qu'inattendues, aussi spontanées que créatives, qui dessinent un monde commun. Dans ces formes d'organisations émergentes, les territoires ruraux font souvent figure d'inspirateur qui bouleverse les relations traditionnelles de dépendance à la ville et innovent dans des synergies renouvelées entre urbain et rural. Ce sont ces signaux faibles de processus en cours qu'il nous faut capter et comprendre pour mieux les accompagner. 


\subsection{Les 3i d'une nouvelle ingénierie territoriale : interterritorialité, intégration et innovation}

Dans une vision d'ensemble de l'ingénierie territoriale, je propose de reprendre le schéma du territoire de projet, à l'articulation entre incitations institutionnelles et initiatives des acteurs locaux, en intégrant trois processus de transformation des territoires (Lardon, 2017) :

- Inter-territorialité (Vanier, 2008): Prendre en compte les liens avec les territoires voisins, l'imbrication dans des échelles plus vastes, la reconnaissance des différenciations territoriales pour ne pas figer un territoire dans ses frontières, mais pour l'ouvrir au monde;
- Intégration territoriale: L'action publique locale met en œuvre elle-même des initiatives qui irriguent les territoires et donnent une nouvelle force à l'action territoriale, et lui apporte une légitimité auprès des institutions. Ce changement des modalités de l'action publique prend tout son sens actuellement dans les projets alimentaires territoriaux, largement déclinés à l'échelle locale et articulés à l'échelle globale;

- Innovation territoriale: Les créations de la société civile débordent les prédictions et inventent « par le bas » de nouvelles visions d'avenir. Les acteurs locaux n'hésitent plus à s'approprier les enjeux territoriaux pour agir au nom du « bien commun » et de valeurs universelles (Magnaghi, 2014). De nouvelles modalités d'action collective telles que start-up de territoire, fab lab ou living lab mobilisent les capacités de tous les acteurs pour développer des idées audacieuses pour le territoire.

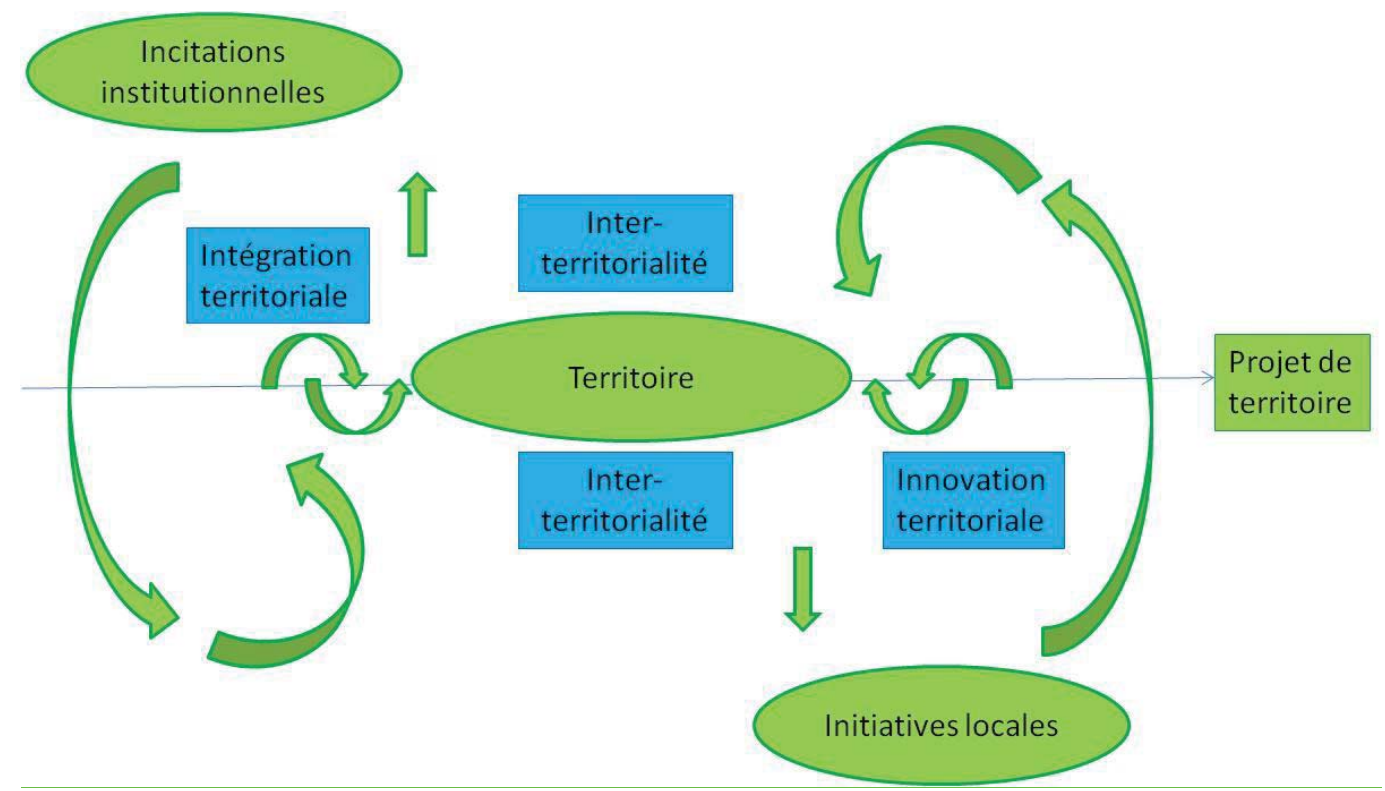

Figure 7 - Les trois processus d'une nouvelle ingénierie territoriale

Ce cycle d'interactions part du territoire, mobilise les capacités locales au nom de valeurs globales, légitime l'intervention des acteurs locaux et des institutions au niveau local et produit de nouvelles dynamiques territoriales. Le problème n'est plus de savoir si l'État insuffle un développement top-down et si les acteurs locaux s'essoufflent à promouvoir un développement bottom-up. Les synergies sont inversées dans la mesure où les institutions publiques ont le pouvoir de soutenir localement les projets et où la société civile a le pouvoir de donner du sens aux projets. Ce renversement des dynamiques engendrées par l'aménagement des territoires n'est-il pas la condition pour inventer nos territoires de demain? (Lardon, 2017, p. 84) 


\subsection{Les 3 vi-e-s d'une nouvelle ingénierie territoriale : vouloir, vivre, voir}

À la lumière des observations récentes, le schéma se complexifie, mais donne les clés de lecture pour accompagner ces processus en impliquant l'ensemble des acteurs qui sont partie prenante. Chaque territoire est responsable de son propre avenir, mais il s'intègre dans une cohérence d'ensemble, car les acteurs conjuguent leurs capacités d'action et les mettent en synergie. Ce qui fait monde commun, ce sont les libres choix que les acteurs font pour dessiner leur territoire de demain en valorisant leurs ressources et leurs compétences. Cela définit les trois vi-e-s des territoires, qu'il s'agit d'entretenir et d'accompagner (voir Figure 8).

\section{Vi comme vision, c'est-à-dire vouloir}

Vouloir se construire une vision partagée des enjeux du territoire et mettre en œuvre des actions collectives ne se fait pas sans les acteurs publics locaux, qui portent les procédures et les dispositifs. Cela ne se fait pas non plus sans les acteurs économiques du marché ni sans les acteurs de la société civile, dans une gouvernance territoriale inclusive (Billion, 2018). Il y a encore du chemin à faire pour une démocratie participative, mais elle ne se fera pas par exclusion des élus, des entreprises ou des militants.

\section{Vie comme vie, c'est-à-dire viorre}

Pour ne pas choisir entre le local et le global, qui entraînent chacun des dérives identitaires ou mondialisantes, Latour (2017) nous propose de choisir un troisième pôle, qu'il appelle «le terrestre», mais qu'on pourrait nommer « territoire de vie ». Il s'agit bien de s'ancrer, "d'appartenir à un sol, vouloir y rester, maintenir le soin d'une terre, s'y attacher » (Latour, 2017, p. 71). "C'est que le terrestre tient à la terre et au sol, mais il est aussi mondial, en ce sens qu'il ne cadre avec aucune frontière, qu'il déborde toutes les identités. » (p.72)

\section{Vis comme visible, c'est-à-dire voir et donner à voir}

Les politiques se territorialisent, mais ont du mal à voir ce qui se passe dans les territoires. Les acteurs institutionnels impulsent des procédures et des dispositifs, mais intègrent difficilement les innovations sociales qui émanent du territoire. Et si on les donnait à voir, si on les rendait visibles et si on se construisait des représentations universelles, non pas parce qu'elles sont uniques, mais parce qu'elles ont du sens dans divers espaces et pour différents acteurs? Elles montreraient le caractère hybride des territoires et contribueraient à l'hybridation des mondes (Gwiazdzinski, 2016).

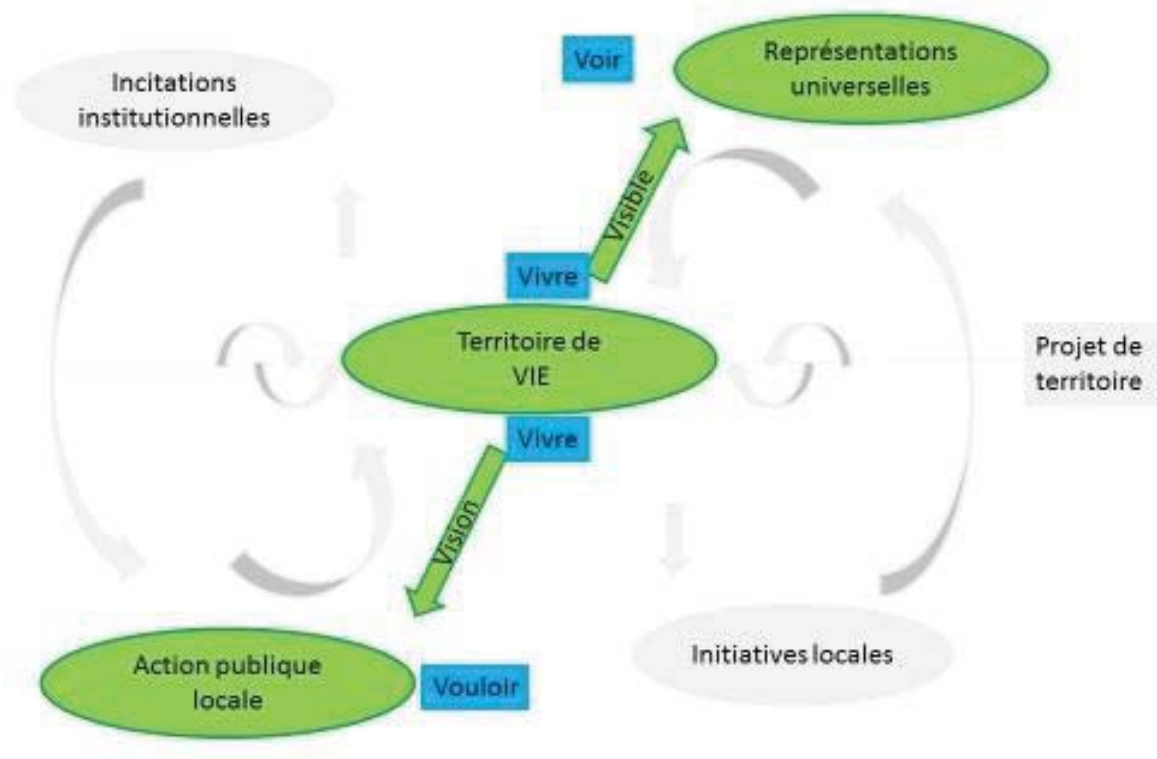

Figure 8 - Les 3 actions d'une nouvelle ingénierie territoriale 
Sans effacer les dynamiques préexistantes, trois nouvelles exigences apparaissent pour accompagner le développement territorial. Assumer que le territoire est un territoire de vie, accepter de le rendre vivable pour tous et accepter de le rendre vivant pour les générations actuelles et futures, c'est là une première exigence. La deuxième exigence est d'entraîner l'action, de la vouloir, dans une vision partagée des enjeux aussi bien locaux que globaux, individuels que collectifs. La troisième exigence est celle de donner du sens à l'action, de construire une représentation d'un monde commun, de rechercher les valeurs universelles et d'en débattre. Cela ne gomme pas les conflits, les hiérarchies ni les pouvoirs; cela n'arrête pas les guerres, les catastrophes naturelles ni les épidémies, mais cela nous rend responsables du futur de notre planète.

Le territoire du Témiscamingue, au Québec, a anticipé ces dynamiques il y a plus de 10 ans lors de la révision de son plan stratégique de gestion. Le jeu de territoire réalisé en 2008 (Lardon, Angeon, Trognon et Leblanc, 2010) a montré des articulations inédites entre les cinq secteurs et structures urbaines du territoire; des solidarités assumées entre les activités agricoles, forestières et touristiques et entre les espaces; des frontières dépassées avec ses relations privilégiées avec l'Ontario, province voisine. Le territoire était bien un territoire de vie, où les acteurs locaux exprimaient leur attachement au territoire et où ils s'adressaient aux acteurs institutionnels pour qu'ils s'engagent dans le développement territorial, sans perdre ni leur authenticité ni leurs valeurs communautaires. Il fait figure de précurseur.

\section{Conclusion : inventons nos territoires de demain}

Dans cette ingénierie territoriale, les chercheurs ont un rôle à jouer pour inlassablement comprendre, expliquer et interpréter les dynamiques, visibles ou invisibles, des territoires, qu'ils soient ruraux ou urbains.

Il y a là de nouvelles pistes à explorer qui peuvent remettre en cause les procédés traditionnels de production scientifique et de construction de l'action. À l'heure où la nécessité de changement devient une évidence pour tous, que ce soit pour les transitions énergétiques, alimentaires ou climatiques; où les institutions connaissent des recompositions qui bousculent les habitudes; et où la société civile prend le pas sur les institutions pour gérer le développement territorial, il est temps de changer nos façons de faire. Loin de penser que la recherche doit répondre aux besoins des acteurs ou que les acteurs doivent contribuer aux recherches comme c'est la conception traditionnelle dans la recherche-action ou en sciences participatives, notre démarche met au cœur les interactions entre chercheurs et acteurs, avec des artefacts qui servent d'objets intermédiaires de représentation et de médiation (Vinck, 1999). Cela permet non seulement de reconnaître et d'intégrer le savoir des acteurs en les hybridant avec le savoir des chercheurs, mais aussi aux chercheurs de pleinement jouer leurs rôles de formalisateurs et de générateurs de connaissances.

La créativité est aussi une qualité qui contribue à une analyse systémique. La science a ainsi à apprendre de l'art pour des démarches plus innovantes. Il importe de valoriser expérience et savoirfaire pour faire évoluer les pratiques des institutions et des individus. La rigueur des itinéraires méthodologiques construits et la diversité assumée des dispositifs permettent de monter en généricité. L'imagination valorise cette diversité et produit le futur des territoires.

\section{NOTES}

1 www6.inra.fr > psdr-inventer

2 www.plate-forme21.fr

3 www.caprural.org

4 https://umr-territoires.fr (La publication est prévue pour 2020 aux Presses universitaires Blaise Pascal de l'Université ClermontAuvergne.)

5 https://agriculture.gouv.fr/programme-national-pour-lalimentation-2019-2023-territoires-en-action 
6 https://adscb05.org/lemediadesacteurs

7 https://caue27.fr

8 https://vallees-habitees.fr/vallee-de-la-levriere-une-belle-mobilisation-pour-le-forum-des-projets

\section{RÉFÉRENCES}

Angeon, V. et Lardon, S. (2003). Dessiner et comprendre le territoire : quand le jeu devient un processus collectif d'apprentissage et de création. Dans B. Debarbieux et S. Lardon S. (dir.), Les figures du projet de territoire (p. 245-257). La Tour-d'Aigues, France : Éditions de l'Aube.

Beauseroy, O., Mabon, M.-Z., Mariotti, S., Lalanne, L. et Lardon, S. (2019, juillet). Jardins potagers, jardins partagés, lieux d'échanges et d'apprentissage collectif. Communication présentée lors du 56e Colloque de l'ASRDLF, Iasi, Roumanie.

Billion, C. (2018). Rôle des acteurs du commerce et de la distribution dans les processus de gouvernance alimentaire territoriale (Thèse de doctorat non publiée). École doctorale LSHS, Université Clermont Auvergne, Clermont-Ferrand, France.

Bletterie, X. et Lardon, S. (2019, juillet). Habitudes alimentaires et forme d'organisation territoriale innovante dans le PNR Queyras. Communication présentée lors du 56e Colloque de l'ASRDLF, Iasi, Roumanie.

Bonnard, J. (2015). Découvrir le monde des objets : former des chercheurs dès l'école maternelle. Lyon, France : Éditions de la Chronique sociale.

Caron, P. (2017). Entre promesses et risques, l'usage du mot territoire dans la pensée du développement agricole. Dans P. Caron, É. Valette, T. Wassenaar, G. Coppens d'Eeckenbrugge et V. Papazian (dir.), Des territoires vivants pour transformer le monde (p. 15-22). Versailles, France : Quæ.

Caron, P., Valette, É., Wassenaar, T., Coppens d'Eeckenbrugge, G. et Papazian, V. (dir.) (2017). Des territoires vivants pour transformer le monde. Versailles, France : Quæ.

CESER Bourgogne-Franche-Comté (2017). Dynamiques territoriales : quelles combinaisons gagnantes? Repéré à http:/ / cgtbourgognefranchecomte.fr/wp-content/uploads/2017/10/Synthèse-Dynamiques-Territoriales-1.pdf

Copello, L. (2019). Mise en perspective du rôle d'une société d'économie mixte et société d'aménagement régional (SEM-S AR) dans le développement territorial (Thèse professionnelle non publiée). AgroParisTech, Paris, France.

Debarbieux, B. et Lardon, S. (dir.). (2003). Les figures du projet de territoire. La Tour-d'Aigues, France : Éditions de l'Aube.

Debarbieux, B. et Vanier, M. (2012). Ces territorialités qui se dessinent. La Tour-d'Aigues, France : Éditions de l'Aube.

Deffontaines, J.-P., Marcelpoil, E. et Moquay, P. (2001). Le développement territorial : une diversité d'interprétations. Dans S. Lardon, P. Maurel et V. Piveteau (dir.), Représentations spatiales et développement territorial (p. 39-56). Paris, France : Hermès.

De la Soudière, M. (2017). Arpenter le paysage : poètes, géographes, montagnards. Paris, France : Anamosa.

Depraz, S. (2017). Penser les marges en France : l'exemple des territoires de «l'hyper-ruralité ». Bulletin de l'Association des géographes francais, 94(3), 385-399. https://doi.org/10.4000/bagf.2086

Direction régionale de l'alimentation, de l'agriculture et de la forêt de la région Auvergne-Rhône-Alpes (DRAAF Auvergne). (2015). Repères et outils pour construire votre Projet Alimentaire Territorial. Repéré à http:/ /draaf.auvergne-rhone-alpes.agriculture.gouv.fr/IMG/pdf/Reperes_et_outils_pour_construire_votre_Projet_Alimentaire_Territorial_cle03d135.pdf

Filippini, R. (2015). Food production potential of periurban agriculture: Contribution of periurban farms to local food system (Thèse de doctorat non publiée). Scuola Superiore Sant'Anna et AgroParisTech, Pise (Italie) et Paris (France).

Gwiazdzinski, L. (dir.). (2016). L'bybridation des mondes : territoires et organisations à l'épreuve de l'bybridation. L'innovation autrement. Grenoble, France : Elya.

Houdart, M. et Nguyen Ba, S. (dir.). (2018). Les réseaux d'acteurs au service de la transition alimentaire : enseignements tirés de la Doume, monnaie citoyenne puydomoise. Paris, France : INRA. Repéré à www6.inra.fr/psdr-inventer/Menu/Les-initiatives-alimentaires/La-Doume

Iceri, V. (2019). Actions collectives alimentaires en territoires ruraux : un regard sur la diversité, une quête pour le développement du territoire. Regard croisé entre Brésil et France (Thèse de doctorat non publiée). École doctorale LSHS, Université Clermont Auvergne, Clermont-Ferrand, France.

Institut national de la recherche agronomique (INRA). (2015). PSDR INVENTER. Paris : INRA. 
Lardon, S. (2013). Le « jeu de territoire », un outil de coordination des acteurs locaux. Revue FaçS ADe, Résultats de recherches du département Inra-Sad, 38 .

Lardon, S. (2017). L'aménagement du territoire au prisme des transitions territoriales : un triple processus à l'œuvre. Pouvoirs locaux, 110(II), 81-86.

Lardon, S., Albaladejo, C., Allain, S., Cayre, P., Gasselin, P., Lelli, L. et Theau, J.-P. (2015). Dispositifs de recherche-formation-action pour et sur le développement agricole et territorial. Dans A. Torre et D. Vollet (dir.), Partenariats pour le développement territorial (p. 47-57). Versailles, France : Qux. https://doi.org/10.3917/quae.torre.2015.01.0047

Lardon, S., Angeon, V., Trognon, L. et Leblanc, P. (2010). Usage du « jeu de territoire » pour faciliter la construction d'une vision partagée du territoire dans une démarche participative. Dans D. Ricard (dir.), Développement durable des territoires : de la mobilisation des acteurs aux démarches participatives (p. 129-145). Ceramac n² 28. Clermont-Ferrand, France : Presses universitaires Blaise Pascal.

Lardon, S., Copello, L. et Albaladejo, C. (2019a, juillet). Regards croisés sur la dynamique de l'élevage dans le territoire de Chascomús : une démarche prospective franco-argentine. Communication présentée lors du 56 Colloque de l'ASRDLF, Iasi, Roumanie.

Lardon, S. et Loudiyi, S. (2014). Agriculture urbaine et alimentation : entre politiques publiques et initiatives locales. Géocarrefour, 89(1-2), 3-10. Repéré à http://journals.openedition.org/geocarrefour/9362

Lardon, S., Marraccini, E., Rizzo, D. et Benoît, M. (2019b, novembre). Combiner cartes à dires d'acteurs et lectures de paysage pour analyser les circuits agricoles et alimentaires de la V allée de la Levrière : un itinéraire méthodologique innovant. Communication présentée lors du Colloque SAGEO, Clermont-Ferrand, France.

Lardon, S., Moquay, P. et Poss, Y. (dir.). (2007). Développement territorial et diagnostic prospectif: réflexions autour du viaduc de Millau. La Tour-d'Aigues, France : Éditions de l'Aube.

Latour, B. (2017). Où atterrir? : Comment s'orienter en politique. Paris, France : La Découverte.

Lièvre, P., Johany, F. et Nguyen Ba, S. (2019, janvier). Les clés des controverses dans PSDR INV ENTER [Fichier vidéo].

Loudiyi, S. et Houdart, M. (2019). L'alimentation comme levier de développement territorial? : les cas de la fête de la Pomme de Massiac et du projet alimentaire territorial du Pays de Courpière. Auvergne, France : Économie rurale.

Magnaghi, A. (2014). La biorégion urbaine : petit traité sur le territoire bien commun. Paris, France : Eterotopia.

Margetic, C., Roth, H. et Pouzenc, M. (2017). Introduction. Dans C. Margetic, H. Roth et M. Pouzenc (dir.), Les campagnes européennes : espaces d'innovation dans un monde urbain (p. 7-11). Toulouse, France : Presses universitaires du Midi.

Ostrom, E. (2009). A general framework for analyzing sustainability of social-ecological systems. Science, 325(5939), 419-422. https://doi.org/10.1126/science.1172133

Parcs naturels régionaux de France (PNR). (2007). Comment une autre vie s’invente ici. Parcs, 58/59.

Torre, A. et Wallet, F. (2015). Towards new paths for regional and territorial development in rural areas. European Planning Studies, 23(4), 650-677. https://doi.org/10.1080/09654313.2014.945812

Trimech, A. et Lardon, S. (2017a). Jeux de territoire 2016 : Avec le PNR Livradois-Forez et le Grand Clermont, inventons nos territoires de demain ». Les 4 pages PSDR INVENTER. Repéré à www6.inrae.fr/psdr-inventer/content/download/3591/35455/version/1/file/Plaquette_JdeT_INVENTER_PNRLF_GC.pub.pdf

Trimech, A. et Lardon, S. (2017b). Jeu de territoire 2017 : Ensemble avec Billom Communauté, inventons notre alimentation de demain. Les 4 pages PSDR INVENTER. Repéré à www.reseaurural-auvergne.fr/wp-content/uploads/2017/10/PLAQUETTE_Billom_communaut\%C3\%A9_2017-.pdf

Valette, É., Caron, P., Coppens d’Eeckenbrugge, G. et Wassenaar, T. (2017). Conclusion générale et perspectives. Dans P. Caron, É. Valette, T. Wassenaar, G. Coppens d'Eeckenbrugge et V. Papazian (dir.), Des territoires vivants pour transformer le monde (p. 263-273). Versailles, France : Qux.

Vanier, M. (2008). Le powvoir des territoires : essai sur linterterritorialité. Paris, France : Economica.

Vinck, D. (1999). Ingénieurs au quotidien : ethnographie de l'activité de conception et d'innovation. Grenoble, France : PUG. 